\title{
The Role of Anthropometric Parameters on Single-Leg Balance Performance in Young Sub-Elite Soccer Players
}

\author{
Dirk Krombholz, Luca Daniel, and \\ Thomas Muehlbauer \\ Peter Leinen \\ University of Duisburg-Essen \\ Saarland University
}

\section{Stefan Panzer \\ Saarland University}

\begin{abstract}
The main purpose of this study was to determine the covariation of anthropometric parameters and the center of pressure $(\mathrm{CoP})$ of young soccer players. Subelite young male players between 16 and 17 years $(N=42)$ were instructed to perform single-leg balance tasks under different conditions: static and dynamic balance on firm and foam ground. Single-leg balance was measured with a Kistler force plate. The measures of postural control were the CoP displacement in anterior-posterior and medio-lateral directions. Further, the following anthropometric variables were assessed: body height, body weight, foot length, and foot width. Results indicated only two small-sized correlations between body height/ weight and the $\mathrm{CoP}$ measures. The covariation between body height, body weight, and the CoP measures for the single-leg stance in young male sub-elite soccer players was less than $10 \%$.
\end{abstract}

Keywords: anthropometry, center of pressure, postural control, youth

A large percentage of our learned movement repertoire involved in everyday activities (e.g., standing, walking) and in sports skills (e.g., kicking, running, skating) requires balance performance (Paillard, 2019; Winter, 1995). Recent findings indicated that balance performance is important to enhance the learning of sports skills (Paillard, 2017 for a review). In addition, Chiari, Rocchi, and Cappello (2002) proposed that balance performance is influenced by biomechanical parameters. To identify relevant biomechanical parameters, namely anthropometric variables, research is necessary that investigates their

Krombholz, Daniel, Leinen, and Panzer are with the Institute of Sport Science, Saarland University, Saarbrücken, Germany. Muehlbauer is with the Department of Sport and Human Movement Science, University of Duisburg-Essen, Essen, Germany. Panzer (s.panzer@mx.uni-saarland.de) is corresponding author. 
relationship to measures of postural control. If there is an association, protocols for the assessment of balance performance have to be adapted to control for these factors. Further, practitioners such as clinicians or coaches can use this information when designing balance training regimes. Recent research from Chiari and colleagues provided experimental evidence that in a bipedal stance, anthropometric parameters such as body height, body weight, and foot width had an impact on balance control when the postural sway was evaluated through Center of Pressure (CoP) measures. This led the researchers to suggest that a few anthropometric parameters are needed to describe the morphology and the individual's balance performance and that some of these parameters can explain up to $50 \%$ of the variation of $\mathrm{CoP}$ measures. Therefore, they concluded that these parameters should be included in the experimental protocol to determine their possible influence on postural control. With a few exceptions (Chiari et al., 2002; Plandowska, Lichota, \& Górniak, 2019), the majority of the studies in the field of balance performance where the researchers collected anthropometric data did not include these data in their analyses. For example, to determine performance asymmetry in leg stability, single-leg balance performance is evaluated. In some studies, balance performance was compared between the dominant and non-dominant leg of children, young adults, and adolescents (e.g., Leinen, Muehlbauer, \& Panzer, 2019; Muehlbauer, Schwiertz, Brueckner, Kiss, \& Panzer, 2019; Snyder \& Cinelli, 2020; Teixeira \& Teixeira, 2008) without consideration of analyzing anthropometric parameters. Young adult individuals are especially characterized by large inter-individual differences in their anthropometric characteristics resulting in accelerated and retarded performers (Musch \& Grondin, 2001). Until now, the existing literature fails to resolve the question of whether anthropometric parameters have an impact on single-leg balance performance in skilled youth athletes. Thus, the main purpose of the present study was to determine the impact of the anthropometric parameters body weight, body height, foot length, and foot width in singleleg stance on the CoP of young sub-elite male soccer players. Extrapolating the results from Chiari et al. (2002) to single-leg balance performance, we expected an impact of the aforementioned anthropometric parameters on $\mathrm{CoP}$ measures.

\section{Methods}

\section{Participants}

Forty-two young male sub-elite athletes were recruited from a soccer club. To determine the dominant foot, all participants had to kick a ball placed on the floor. Seven athletes kicked the ball with the left foot. All soccer players played at the highest or second highest league for their age. Systematic soccer playing experience ranged from 11 to 12 years. Descriptive data of the anthropometry is presented in Table 1 . None of the participants had an injury at the lower extremities during the previous three months. Informed consent approved by the local ethics committee was provided prior to participation by all participants and their parents. The study was conducted in accordance with the revised version of the 1964 Declaration of Helsinki. 


\section{Table 1 Descriptive Data of the Study Participants ( $M$; in Parentheses Range: Minimum-Maximum)}

\begin{tabular}{lccccc}
\hline $\begin{array}{l}\text { Male } \\
\text { Soccer } \\
\text { Players }\end{array}$ & $\begin{array}{c}\text { Age } \\
\text { (years) }\end{array}$ & $\begin{array}{c}\text { Body } \\
\text { Height } \mathbf{( c m )}\end{array}$ & $\begin{array}{c}\text { Body } \\
\text { Mass } \mathbf{( k g )}\end{array}$ & $\begin{array}{c}\text { Foot Length } \\
\mathbf{( m m})\end{array}$ & $\begin{array}{c}\text { Foot Width } \\
\text { (mm) }\end{array}$ \\
\hline$N=42$ & 16.4 & 177.3 & 66.7 & Left: 263.6 & Left: 100.3 \\
& $(16-17)$ & $(166.0-187.0)$ & $(52.5-87.4)$ & $(240.3-291.0)$ & $(89.4-112.3)$ \\
& & & & Right: 262.7 & Right: 100.8 \\
& & & & $(240.3-287.5)$ & $(90.4-111.8)$ \\
\hline
\end{tabular}

\section{Apparatus and Tasks}

The measurement system used for the balance testing consisted of a force plate (Kistler 9286AA; Winterthur, Switzerland) and a computer (HP 1040 G4, Palo Alto, CA, USA). With a Matlab 2019 (version 2019a; The MathWorks, Natick, MA) script and the data acquisition tool box, the ground reaction forces were recorded (sampling rate: $333 \mathrm{~Hz}$ ) and stored on a computer for later offline analysis. Participants were instructed to perform eight different tasks with eyes open. The foot position of the force plate was standardized by two markers positioned $15 \mathrm{~cm}$ left and $15 \mathrm{~cm}$ right from the midline of the force plate. The inner foot was placed on the left marker when the left foot was the stance foot, and on the right marker when the right foot was the stance foot. As in the Leinen et al. (2019) protocol, tasks 1 to 4 represent the static and tasks 5 to 8 the dynamic balance tasks, respectively. The four static balance tasks included the single-leg stance with the right and left leg on firm and foam ground. The four dynamic balance tasks consisted of the single-leg stance with the right and left leg on firm and foam ground with the extended contralateral leg swinging back and forth by hip flexionextension. During all balance tasks, participants were instructed to stand barefoot on the force plate with their hands placed on their hip and to fixate on a circle (10 cm diameter) two meters in front of them at eye-level to avoid head movements. The static balance tasks were timed for $30 \mathrm{~s}$ and the dynamic ones were measured for $6 \mathrm{~s}$. Movement amplitude for the dynamic balance tasks was set at $40 \mathrm{~cm}$ indicated by two markers $20 \mathrm{~cm}$ in front of and $20 \mathrm{~cm}$ behind the athletes. A metronome $(2 \mathrm{~Hz}$ ) was used to pace the oscillatory movements for the dynamic balance tasks. The static and dynamic balance tasks were counterbalanced to reduce the influence of task order. The firm ground was the surface of the force plate and the foam ground was a balance-pad from AIREX company (Sins, Switzerland; thickness: $6.5 \mathrm{~cm}$ ) positioned on top of the platform with the two markers on top of the pad. Foot anthropometry was measured by a $3 \mathrm{~A}$ flatbed scanner (SN GP-FSC-2008-102-P; Germany) with a spatial resolution of $600 \times 850$ pixels. The athletes were instructed to step on the scanner and to position their feet into a self-selected side-by-side stance and not to move as long as their feet were traced by the scanner. Foot length was assessed as the length of the distal end of the toe to the midpoint of the heel. Foot width was defined as the widest foot perpendicular to the orientation of the foot length line (see Chiari et al., 2002). 


\section{Procedure}

The testing was performed individually in a quiet room. Written instructions were provided to all athletes. The following testing sequence was applied. Assessment of (a) the dominant leg, (b) body height in cm using a stadiometer (seca 213, Basel, Switzerland), (c) foot anthropometry (i.e., foot length and width in $\mathrm{mm}$ ), (d) body weight in $\mathrm{kg}$ using the force plate (calculated from the ground reaction forces in $\mathrm{Fz}$ direction), and (e) static and dynamic balance. If an athlete lost balance control during a trial, the trial was repeated ( $4.5 \%$ of the trials had to be recorded again). Between the balance tasks, the participants had a rest interval of approximately 1 minute. This was done to reduce fatigue. During this time interval, the data of the trial were saved.

\section{Data Analysis}

Data analyses were performed using Matlab 2019a. To evaluate balance performance, the individual trial time series of the ground reaction forces were used to calculate the CoP displacements. To reduce noise in the data, the force time series of each trial was filtered with a low-pass filter (2nd order dualpass Butterworth filter) with a cutoff-frequency of $10 \mathrm{~Hz}$. Balance performance was assessed by a two-dimensional (2D) measure. The 2D measure was the standard deviation $(S D)$ of the CoP displacement $(\mathrm{mm})$ in the anterior-posterior and the medio-lateral directions (Winter, 1995). Statistical analyses were conducted with SPSS 25.0 (IBM Corp., Armonk, NY). According to Chiari et al. (2002), who identified body weight, body height, and foot width as significant anthropometric parameters, Pearson product-moment correlation was used to assess if these variables co-varied with the $\mathrm{CoP}$ displacement in the anteriorposterior and medio-lateral directions for the dominant and the non-dominant leg. In addition, foot length was included and the determination coefficient $r^{2}$ was calculated for the significant correlations. To determine the linear dependence of the anthropometric parameters and the CoP displacements in anteriorposterior and medio-lateral direction, the data were analyzed with a linear regression analysis.

\section{Results}

The results of the Pearson correlation $r$ and the regression slope, $B$, are provided in Table 2. Examples of the CoP displacement in the 2D horizontal plane from the non-dominant leg of a soccer player with low body weight and a player with high body weight in the static condition on firm ground are provided in Figure 1a and 1b. Figure 1c and 1d illustrate the CoP displacements in anterior-posterior and medio-lateral directions for the non-dominant leg of a small and a tall soccer player in the dynamic condition on firm ground.

These data illustrate that the soccer player with a high body weight compared to a soccer player with a low body weight showed a larger displacement of the CoP in anterior-posterior direction for the non-dominant leg in the static condition on firm ground, while the displacement of the CoP in medio-lateral direction did not differ between the two players (Figure 1a and 1b). 


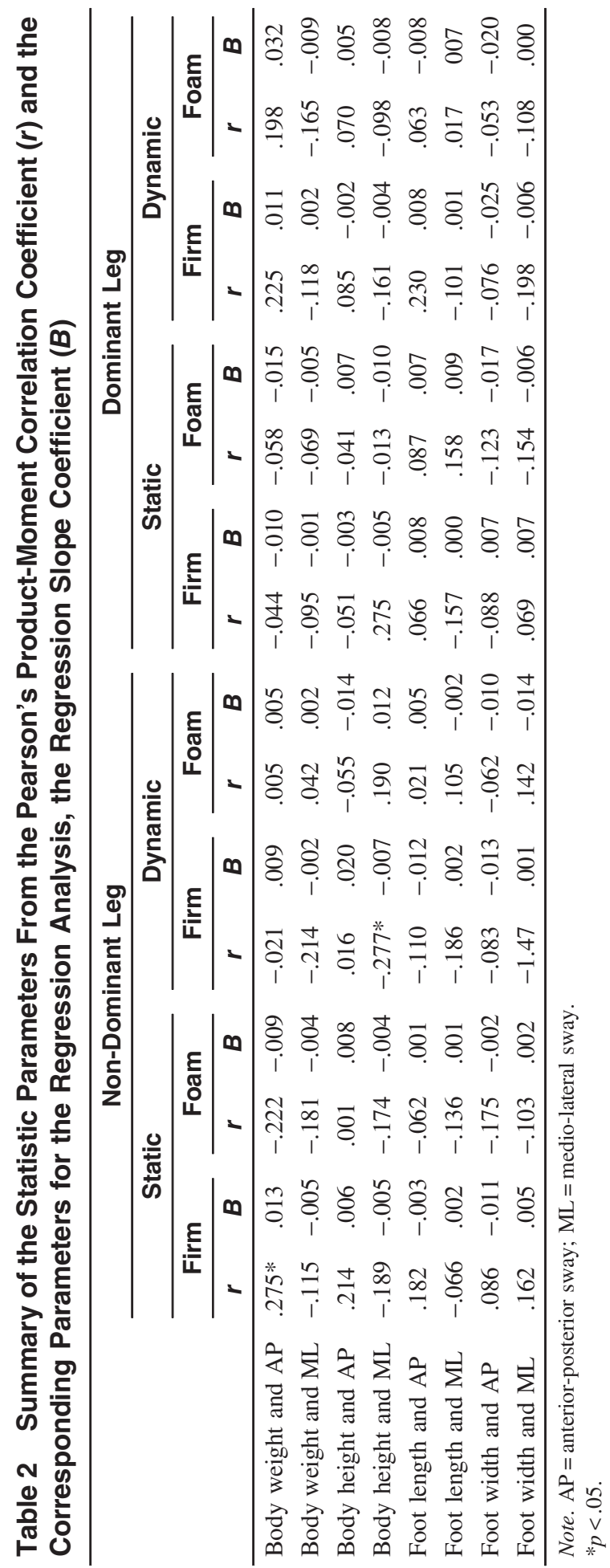



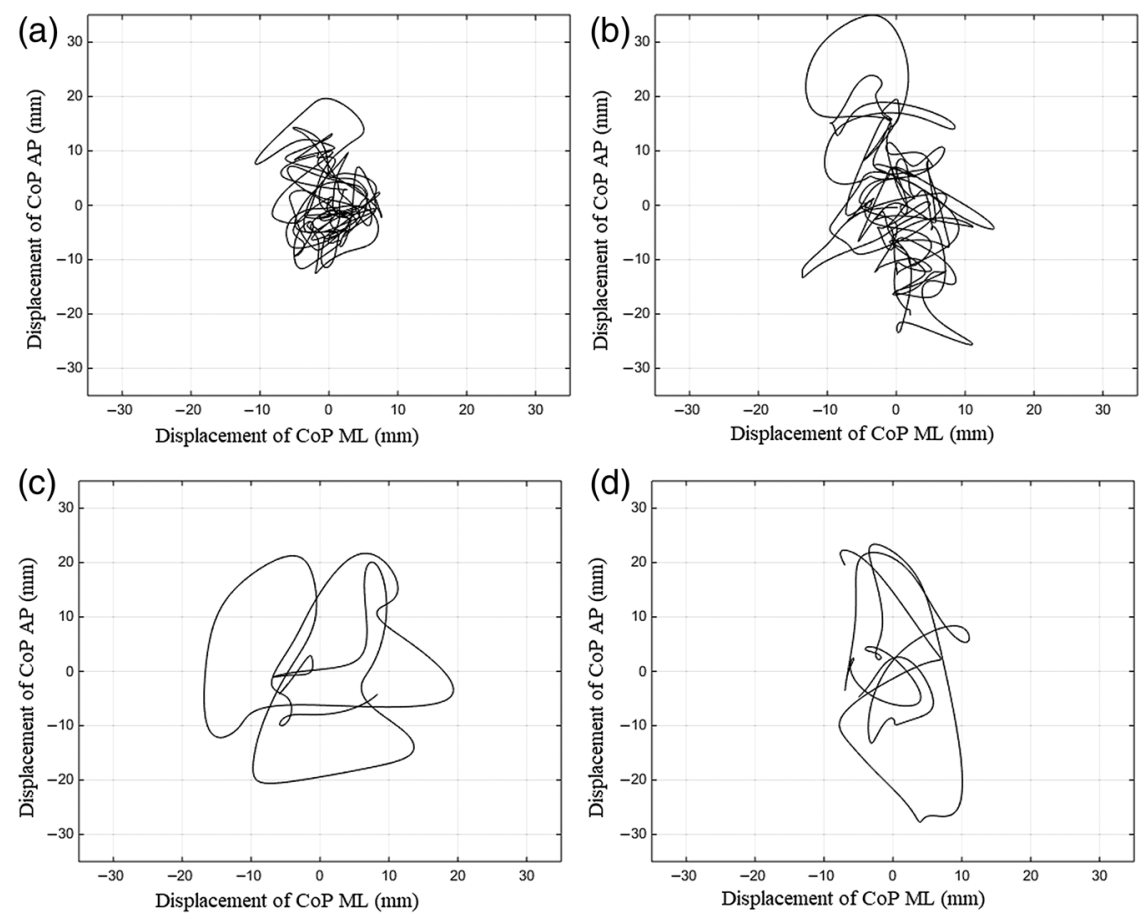

Figure 1 - Examples of individual center of pressure $(\mathrm{CoP})$ displacements in anteriorposterior (AP) and medio-lateral (ML) direction. Figure $1 \mathrm{a}$ and $1 \mathrm{~b}$ illustrate the CoP displacement in AP and ML directions from the non-dominant leg of a soccer player with low (a) and high (b) body weight in the static condition on firm ground. Figure 1c and 1d illustrate the $\mathrm{CoP}$ displacements in AP and ML directions from the non-dominant leg of a small (c) and a tall (d) soccer player in the dynamic condition on the firm ground.

Further, the CoP displacements in Figure 1c and 1d showed that the displacement of the $\mathrm{CoP}$ in the medio-lateral direction on the non-dominant leg for the dynamic condition on firm ground was smaller for the tall player than for the small player while the displacement in anterior-posterior direction did not differ between both players. The individual examples indicated that athletes with the different body morphology produced CoP displacement patterns which coincides with our correlation analyses. The results of the correlation analyses indicated only two significant co-variations: (1) body weight significantly co-varied with the $S D$ of the CoP in anterior-posterior direction for the non-dominant leg in the static condition on firm ground and (2) body height significantly co-varied with the $S D$ of the CoP in medio-lateral direction for the non-dominant leg in the dynamic condition on firm ground. The first coefficient was positive, which indicated that an increase in body weight resulted in an increase in $S D$ of the CoP. The latter one was negative which indicated that an increase in body height resulted in a decrease in $S D$ of the CoP. The determination coefficient for the first correlation was $r^{2}=.075$ and for the latter was $r^{2}=.077$. This indicated that a percentage of $\sim 8 \%$ of the 
$S D$ of the CoP can be explained by body weight or height. All other correlation coefficients failed to reach significance (see Table 2). The results of the linear regression analysis indicated that the linear dependence of the $S D$ of the CoP and the anthropometric parameter were low. Postural control for the non-dominant and dominant leg was not predicted by one of the anthropometric parameter (i.e., body weight, body height, foot length, or foot width).

\section{Discussion}

The main purpose of the present study was to determine the impact of anthropometric data (body weight, body height, foot length, foot width) on CoP measures while standing on one leg in young sub-elite male soccer players. Therefore, players performed a single-leg stance under a static and dynamic condition on firm and foam ground. The analyses indicated that all correlation values were low and only two correlations were statistically significant. The corresponding slopes of the regression equations were of little predictive value. The preceding results showed only a limited relationship between the anthropometric data of body weight and body height to the CoP measures. The findings that body height and body weight co-varied with postural control on a significant level is consistent with the results reported by Chiari et al. (2002). Although the anthropometric parameter body height is relevant for any inverted pendulum model (Winter, 1995) to explain postural control, the small values of the determination coefficient indicated that the common variance between the two anthropometric parameters body height and weight and the CoP measures in the present study was less than $10 \%$. Foot length and width had no significant influence on postural control. Note, the foot position on the platform was standardized across the test conditions (see Kriby, Price, \& Mac Leod, 1987).

On a closer inspection, the experimental protocol from the previous studies (Chiari et al., 2002; Plandowska et al., 2019), which included anthropometric parameters into the analysis, and the present study differ in some aspects that could explain the discrepancies. Plandowska et al. (2019) tested bipedal postural stability of 5-year-old boys and girls. In the study from Chiari and colleagues, postural control was assessed by bipedal stance with eyes open and eyes closed in healthy and physically active adults. The correlations between the body height, body weight, and foot width significantly co-varied with the postural control parameters under the eyes closed condition. In the present study, participants were young sub-elite soccer players who had up to 12 years of soccer experience with four to five practice sessions a week. Further, we tested postural control during single-leg stance with eyes open. In addition, the mean anthropometric parameters for the male participants in the Chiari et al. (2002) study were $176.6 \mathrm{~cm}$ for body height and $74.8 \mathrm{~kg}$ for body weight. In the present study, the mean body height was $177.3 \mathrm{~cm}$ and the body weight amounted to $66.7 \mathrm{~kg}$. Thus, the difference between our study and that of Chiari and colleagues was less than $1 \mathrm{~cm}$ for body height and less than $10 \mathrm{~kg}$ for the body weight. According to the small differences of the mean anthropometric parameters between the individuals in the two studies, it is not conceivable that the discrepancies between the two studies are due to these different parameters. However, the different relationships between the anthropometric parameters and 
postural control evaluated by $\mathrm{CoP}$ measures could result from (a) the deprivation of the visual system in the Chiari et al. study, (b) the training status, and/or (c) the stance condition (i.e., bipedal stance vs. single-leg stance; foot position) (see Paillard, 2019).

\section{Conclusion and Future Directions}

Our findings demonstrate that in young male sub-elite soccer players balance performance in single-leg stance is only slightly affected by the anthropometric parameters body height and weight. Further, foot length and foot width did not covary with postural control. It is suggested that anthropometric variables have a not exceeding (foot length and width) or only marginal (body weight and height) influence when evaluating postural performance through $\mathrm{CoP}$ measures in young sub-elite male soccer players with single-leg stance in an eyes-open condition. The one-to-one extrapolation of the findings in bipedal stance and anthropometric parameters such as body height and body weight in healthy adults to single-leg balance performance should be considered with caution. Compared to the studies of Chiari et al. (2002) and Plandowska et al. (2019), it should be taken into account that in the current study a special population of successful young athletes participated. They had a lot of soccer experience including single-leg balance performance while passing and kicking the ball. However, the results are specific to the test methodology used to assess balance performance (i.e., SD of COP during single-leg stance). This measure/stance condition may not represent all components of balance performance. Therefore, caution is needed when generalizing the present findings to other test situations. This suggests that the influence of anthropometric parameters on balance performance in different populations, other CoP measures, and different balance tasks need to be more directly examined in future studies.

\section{Acknowledgments}

We would like to thank all the athletes and coaches from SV Darmstadt 98 for their participation in the study. The authors have no conflicts of interest to disclose.

\section{References}

Chiari, L., Rocchi, L., \& Cappello, A. (2002). Stabilometric parameters are affected by anthropometry and foot placement. Clinical Biomechanics, 17(9-10), 666-677. PubMed ID: 12446163 doi:10.1016/S0268-0033(02)00107-9

Kriby, R.L., Price, N.A., \& Mac Leod, D.A. (1987). The influence of foot position on standing balance. Journal of Biomechanics, 20(4), 423-427. PubMed ID: 3597457 doi:10.1016/0021-9290(87)90049-2

Leinen, P., Muehlbauer, T., \& Panzer, S. (2019). Single-leg balance performance in sub-elite young soccer players and swimmers as a function of age and sports experience. Journal of Motor Learning and Development, 7(3), 374-388. doi:10.1123/jmld.2018-0055

Muehlbauer, T., Schwiertz, G., Brueckner, D., Kiss, R., \& Panzer, S. (2019). Limb differences in unipedal balance performance in young male soccer players with different ages. Sports, 7(1), 20. doi:10.3390/sports7010020 
Musch, J., \& Grondin, S. (2001). Unequal completion as an impediment to persona development: A review of the relative age effect in sport. Developmental Review, 21(2), 147-167. doi:10.1006/drev.2000.0516

Paillard, T. (2017). Plasticity and the postural function to sport and/or motor experience. Neuroscience \& Biobehavioral Reviews, 72, 129-152. PubMed ID: 27894829 doi:10. 1016/j.neubiorev.2016.11.015

Paillard, T. (2019). Relationship between sport expertise and postural skills. Frontiers in Psychology, 10, 1428 doi:10.3389/fpsyg.2019.01428

Plandowska, M., Lichota, M., \& Górniak, K. (2019). Postural stability of 5-year-old girls and boys with different body heights. PLoS One, 14(12), e0227119. PubMed ID: 31887198 doi:10.1371/journal.pone.0227119

Snyder, N., \& Cinelli, M. (2020). Comparing balance control between soccer players and non-athletes during a dynamic lower limb reaching task. Research Quarterly for Exercise and Sport, 91(1), 166-171. doi:10.1080/02701367.2019.1649356

Teixeira, M.C.T., \& Teixeira, L.A. (2008). Leg preference and interlateral performance asymmetry in soccer player children. Developmental Psychobiology, 50(8), 799-806. PubMed ID: 18688809 doi:10.1002/dev.20322

Winter, D.A. (1995). Human balance and posture control during standing and walking. Gait \& Posture, 3(4), 193-214. doi:10.1016/0966-6362(96)82849-9 